\title{
Determinação dos padrões de viagens e taxas de geração de viagens de automóveis e caminhões para indústrias: o caso de Tubarão/SC
}

\author{
Mayara Orlandi Silva ${ }^{1}$; Lenise Grando Goldner²
}

\begin{abstract}
Resumo: Assim como outros Pólos Geradores de Viagens (PGVs), as indústrias provocam mudanças no tráfego de seu entorno, devido às viagens realizadas por funcionários e caminhoneiros. Este trabalho apresenta uma pesquisa realizada entre os meses de março e junho de 2008, em três indústrias na cidade de Tubarão - SC, que consistiu em entrevistas com a administração destas indústrias e a aplicação de questionários a uma amostra estratificada de funcionários e caminhoneiros. Também foram realizadas contagens volumétricas de tráfego nos principais acessos. Da análise dos resultados, obteve-se a movimentação da entrada e saída de veículos em geral e a percentagem de pico horário correspondente. Mediante o somatório de fluxos, foi possível elaborar as taxas de geração de viagens em função da área total construída e do número de funcionários. Realizou-se a comparação das taxas e volumes de viagens propostas na pesquisa com os modelos existentes referentes ao tipo de PGV estudado.
\end{abstract}

Abstract: Just like other Trip Generating sites, industries bring about changes in the traffic in their surroundings due to the trips made by employees and truck drivers. This paper describes a research survey conducted from March to June 2008 in three industries in the city of Tubarão - Santa Catarina. It consisted of interviews with top management staff and the administration of questionnaires to a stratified sample of industry employees and truck drivers. Traffic volume counts were conducted in the main access ways and the analysis of the results provided a portrait of the movement of vehicles entering and departing in general and the peak hour percentages of traffic volumes. The sum of flows was used to calculate trip generation rates as a function of gross floor areas and numbers of employees. A comparison was made of the rates and trip volumes obtained with those proposed by existing models for the type of PGV studied.

\section{INTRODUÇÃO}

O crescimento acelerado das cidades brasileiras e da frota de veículos nas últimas décadas, bem como a concentração da população e de atividades econômicas num mesmo espaço, desencadeou dificuldades consideráveis para os municípios e suas administrações, principalmente quanto à segurança e fluidez do tráfego. A expansão urbana desordenada, agravada pela ausência de um planejamento e de estratégias específicas para esse controle, contribuiu para o aumento dos problemas relacionados à ocupação do espaço físico. O uso e a ocupação do solo apresentam reflexos de diversas atividades, entre elas, a industrial, responsável por alterações na qualidade da água, do ar, do solo e pela circulação viária, resultando em congestionamentos e acidentes de trânsito que interferem diretamente na qualidade de vida da população.

Segundo Carlos (1990), para se desenvolver, a indústria pressupõe a concentração espacial, beneficiando-se da infraestrutura e mão de obra local e da proximidade de outras indústrias complementares. A in-

\footnotetext{
${ }^{1}$ Mayara Orlandi Silva, Programa de Pós-graduação em Engenharia Civil, Universidade Federal de Santa Catarina, Florianópolis, SC, Brasil. (e-mail: mayaraeng@hotmail.com).

${ }^{2}$ Lenise Grando Goldner, Programa de Pós-graduação em Engenharia Civil, Universidade Federal de Santa Catarina, Florianópolis, SC, Brasil. (e-mail: lenise@ecv.ufsc.br).
}

Manuscrito recebido em 7/12/2009 e aprovado para publicação em 29/1/2010. Este artigo é parte de TRANSPORTES, volume XVIII, número 1, março de 2010. ISSN: 2237-1346 (online). dústria se aproveita ao máximo dessas vantagens, enquanto socializa as desvantagens como poluição e congestionamentos. As indústrias são empreendimentos de grande porte, também chamados de Pólos Geradores de Viagens (PGVs), pois potencializam a geração de viagens, circulação de pessoas, mercadorias e veículos, causando impactos em diversos âmbitos, principalmente no entorno do empreendimento. Mesmo com a importância desses impactos, o que se pode observar é que os estudos sobre indústrias como PGV são insuficientes no Brasil e bem pouco explorados no exterior, com uma escassa bibliografia. Destacam-se os trabalhos da CET-SP (1983), Keefer (1966) e do ITE (2008), que elaboraram taxas de geração de viagens para esse tipo de empreendimento.

O objetivo do presente artigo é apresentar os resultados de uma pesquisa que analisou as indústrias como Pólos Geradores de Viagens, buscando identificar seus padrões de viagens e elaborar taxas de geração de viagens de automóveis e caminhões, de modo a obter parâmetros que possibilitem avaliar seus impactos no sistema viário e de transportes e, com isto, auxiliar o planejador de transportes a realizar esse tipo de estudo.

Como medida de economia de recursos, a pesquisa foi realizada somente em Tubarão, cidade localizada no sul do Estado de Santa Catarina, devido à presença efetiva dessas edificações, e na qual existem vários parques industriais que atuam em diferentes áreas, principalmente no setor cerâmico, importantes não só 
no contexto econômico estadual, mas também nacional, respondendo a um percentual significativo da capacidade de produção nacional deste tipo de produto.

\section{REVISÃO BIBLIOGRÁFICA}

São escassas as bibliografias relacionadas às indústrias sobre o aspecto da engenharia de tráfego. Um estudo mais recente é o de Pitombo et al. (2006), que investigou a influência do uso do solo e das características socioeconômicas na sequência de deslocamentos dos industriários na Região Metropolitana de São Paulo (RMSP), formada por 389 zonas de tráfego.

Os autores verificaram que a influência das variáveis socioeconômicas (o uso de vale-transporte, a renda familiar e número de automóveis no domicílio) refletiu-se principalmente na escolha dos modos de transporte, sendo que indivíduos com maior renda familiar realizam, predominantemente, suas viagens utilizando automóvel próprio, enquanto aqueles com menor renda utilizam o transporte público. A variável uso do solo relacionou-se predominantemente com a sequência de destinos. A variável proposta no estudo dos autores considera a taxa de indústria acumulada (número de empregos na indústria na zona por número total de empregos na indústria) por raio, a partir do centróide das zonas de tráfego. Tal variável representa o grau de atratividade da zona de origem e a atratividade/oportunidade acumulada por diferentes raios de ação. Conforme os autores, indivíduos que moram em zonas de tráfego com taxa de indústria alta (até um raio de $5 \mathrm{~km}$ ) tendem a realizar viagens curtas ao trabalho, enquanto aqueles que residem em zonas de tráfego com taxa de indústria acumulada baixa tendem a realizar viagens mais longas por motivo de trabalho.

A Companhia de Engenharia de Tráfego - CET (1983) analisou alguns empreendimentos na cidade de São Paulo e divulgou, em seu trabalho, equações de regressão linear que permitem determinar a geração de viagens de pólos geradores do tipo indústria em fun- ção do número de funcionários, área total construída e área total do terreno. As equações 1, 2 e 3 definidas são:

$$
V=0,545 \cdot N F-12,178 \quad, R^{2}=0,875
$$

em que, $V$ : número médio de viagens atraídos na hora de pico para a indústria;

$N F$ : número total de funcionários.

$$
V=0,031 \cdot A C-23,653 \quad, R^{2}=0,684
$$

sendo, $\quad V$ : número médio de viagens atraídos na hora de pico para a indústria;

$A C$ : área construída $\left(\mathrm{m}^{2}\right)$.

$$
V=0,021 \cdot A T-4,130 \quad, R^{2}=0,687
$$

em que, $V$ : número médio de viagens atraídos na hora de pico para a indústria;

AT: área total construída $\left(\mathrm{m}^{2}\right)$.

No exterior, a principal publicação sobre o assunto é do ITE (2008), denominada Trip Generation que apresenta um estudo sobre diversos tipos de PGVs, definindo, entre eles, taxas e modelos de geração de viagens para indústrias, descritos na Tabela 1. Estas taxas e modelos referem-se, portanto, a soma das viagens atraídas e produzidas pelos empreendimentos no período de tempo considerado.

Um estudo americano mais antigo, considerado um dos pioneiros no assunto, é o de Keefer (1966), que determinou a geração de viagens para indústrias em função do número de funcionários para 44 empreendimentos, conforme se apresenta na Tabela 2. Embora seu estudo seja bastante antigo, optou-se por apresentar os dados definidos pelo autor, em razão de não serem encontrados, na bibliografia disponível, estudos mais recentes sobre o tema com o mesmo enfoque. Ressalta-se que estes números provavelmente não representam a realidade atual e devem ser analisados

\begin{tabular}{|c|c|c|c|c|c|}
\hline Categoria & $\begin{array}{l}\text { Uso do } \\
\text { Solo }\end{array}$ & $\begin{array}{c}N^{o} \text { de } \\
\text { Estudos }\end{array}$ & $\begin{array}{c}\text { Unidade ou } \\
\text { variável explicativa } \\
(x) \\
\end{array}$ & $\begin{array}{c}\text { Taxa média de viagens } \\
\text { geradas por unidade de } \\
\text { tempo } \\
\text { (intervalo) }\end{array}$ & $\begin{array}{c}\text { Equaçãóo } \\
\left(R^{2}\right)\end{array}$ \\
\hline \multirow{6}{*}{ Indústria } & \multirow{2}{*}{ Leve } & 21 & $\mathrm{~N}^{\mathrm{o}}$ de empregados & $\begin{array}{c}0,51 / \text { hora pico da tarde } \\
(0,36-1,18)\end{array}$ & $\begin{array}{c}0,358 X+65,81 \\
(0,90) \\
\end{array}$ \\
\hline & & 27 & $\begin{array}{l}1.000 \text { pés}^{2} \text { área } \\
\text { bruta construída }\end{array}$ & $\begin{array}{c}\text { 1,08/hora pico da tarde } \\
(0,36-4,50)\end{array}$ & $\begin{array}{c}1,422 \mathrm{X}-125,200 \\
(0,89)\end{array}$ \\
\hline & \multirow{2}{*}{ Pesada } & 3 & $\mathrm{~N}^{\circ}$ de empregados & $\begin{array}{c}0,40 / \text { hora pico da tarde } \\
(0,22-1,10)\end{array}$ & - \\
\hline & & 2 & $\begin{array}{l}1.000 \text { pés }^{2} \text { área } \\
\text { bruta construída }\end{array}$ & $\begin{array}{c}0,68 / \text { hora pico da tarde } \\
(0,49-0,78)\end{array}$ & - \\
\hline & \multirow{2}{*}{ Manufatureira } & 51 & $\mathrm{~N}^{\mathrm{o}}$ de empregados & $\begin{array}{c}\text { 0,40/hora pico da tarde } \\
(0,24-1,11)\end{array}$ & $\begin{array}{c}0,822 \operatorname{LnX}+0,309 \\
(0,93)\end{array}$ \\
\hline & & 50 & $\begin{array}{l}1.000 \text { pés }^{2} \text { área } \\
\text { bruta construída }\end{array}$ & $\begin{array}{c}\text { 0,78/hora pico da manhã } \\
(0,10-8,75)\end{array}$ & $\begin{array}{c}0,829 X-17,713 \\
(0,81) \\
\end{array}$ \\
\hline
\end{tabular}
com cautela.

Tabela 1. Taxas e modelos de geração de viagens para indústrias americanas 
Tabela 2. Viagens a indústrias selecionadas por grupo de funcionários

\begin{tabular}{|c|c|c|c|c|c|c|c|}
\hline \multirow[b]{2}{*}{$\begin{array}{c}\text { Grupo de } \\
\text { funcionários }\end{array}$} & \multirow[b]{2}{*}{$\begin{array}{c}N^{o} d e \\
\text { indústrias }\end{array}$} & \multicolumn{3}{|c|}{ Veículos de passeio } & \multicolumn{3}{|c|}{ Caminhões } \\
\hline & & $\begin{array}{l}\text { Total de } \\
\text { viagens } \\
\text { diárias }\end{array}$ & $\begin{array}{c}\text { Total de } \\
\text { viagens } \\
\text { pico }\end{array}$ & $\begin{array}{c}\text { Volume } \\
\text { de pico } \\
(\%)\end{array}$ & $\begin{array}{c}\text { Total de } \\
\text { viagens } \\
\text { diárias }\end{array}$ & $\begin{array}{c}\text { Volume } \\
\text { diário } \\
(\%)\end{array}$ & $\begin{array}{c}\text { Volume } \\
\text { de pico } \\
(\%)\end{array}$ \\
\hline $0-999$ & 9 & 5.021 & 1.866 & 37 & 319 & 6,5 & 6,6 \\
\hline $1.000-1.999$ & 12 & 14.107 & 5.235 & 37 & 891 & 6,3 & 10,9 \\
\hline $2.000-2.999$ & 8 & 14.088 & 6.169 & 44 & 499 & 3,4 & 5,0 \\
\hline $3.000-3.999$ & 4 & 9.840 & 4.422 & 45 & 256 & 2,5 & 1,6 \\
\hline $4.000-4.999$ & 6 & 17.465 & 7.129 & 41 & 1.234 & 6,6 & 2,9 \\
\hline $5.000-9.999$ & 4 & 16.024 & 6.622 & 41 & 870 & 5,1 & - \\
\hline $10.000-19.999$ & 1 & 6.730 & 2.423 & 36 & 303 & 4,3 & - \\
\hline Total & 44 & 82.275 & 33.867 & 41 & 4.372 & 5,1 & 4,2 \\
\hline
\end{tabular}

Apresentam-se, a seguir, os principais resultados obtidos no estudo de caso para as indústrias da cidade de Tubarão, em relação aos seus padrões de viagens.

\section{ESTUDO DE CASO}

\subsection{Descrição das indústrias}

As indústrias objeto deste estudo, que não terão seus nomes divulgados devido ao sigilo da pesquisa, serão denominadas por indústria 1, indústria 2 e indústria 3 e caracterizadas na Tabela 3.

$\mathrm{O}$ acesso às indústrias 2 e 3 é realizado pela $\mathrm{BR}$ 101, sendo seu único acesso. A indústria 1 está situada em uma área residencial e extremamente urbanizada, o que ocasiona impactos à BR-101 e também às vias arteriais e locais que lhe dão acesso.

\subsection{Levantamento de dados}

Entre os meses de março e junho de 2008, foi realizado um levantamento de dados, nas indústrias 1 , 2 e 3 , por meio de pesquisas com a administração, funcionários e caminhoneiros, além da contagem volumétrica de tráfego.

Com a administração, obtiveram-se dados sobre os empreendimentos, tais como: produtos fabricados, a produção anual, a área total construída, o número de funcionários e os turnos de trabalho. Na pesquisa com funcionários e caminhoneiros, aplicou-se um total de 317 e 87 questionários respectivamente, em dias típi- cos das indústrias (entre terças e quintas-feiras), com questões referentes às características das viagens à empresa e dos próprios entrevistados: modo de transporte de ida e volta à indústria, local de origem e destino das viagens, tempos de viagem, sexo, idade, escolaridade e renda bruta individual de funcionários e caminhoneiros, a fim de se determinar o perfil socioeconômico e as características das viagens.

Foi obtida uma amostra de 30\% dos funcionários das três empresas. Essa amostra foi do tipo proporcional estratificada, por turno e setor de trabalho, escolhida aleatoriamente para um intervalo de confiança de $95 \%$ e erro amostral entre 7,4\% e 8,6\%. Quanto aos caminhoneiros, utilizou-se $50 \%$ da amostra, para um intervalo de confiança de $95 \%$ e erro amostral entre $2,0 \%$ e $2,10 \%$.

Paralelamente à aplicação dos questionários, realizaram-se pesquisas de campo mediante contagens volumétricas de veículos particulares e caminhões nos acessos às indústrias. A pesquisa contou com a participação de duas pessoas, que anotaram os fluxos de entrada e saída, num período de 24 horas, em intervalos de 15 minutos, também em dias típicos do empreendimento (entre terças e quintas-feiras), e visou à determinação das taxas de geração de viagens por automóveis e caminhões.

Tabela 3. Características das indústrias selecionadas

\begin{tabular}{|c|c|c|c|c|c|c|c|c|c|}
\hline \multirow[b]{2}{*}{ Ind. } & \multirow[b]{2}{*}{ Tipo } & \multirow[b]{2}{*}{ Mercado } & \multirow{2}{*}{$\begin{array}{l}\text { Volume } \\
\text { produção }\end{array}$} & \multirow[b]{2}{*}{ Produto } & \multirow{2}{*}{$\begin{array}{l}A C \\
\left(m^{2}\right)\end{array}$} & \multicolumn{2}{|c|}{ Funcionários } & \multicolumn{2}{|c|}{ Caminhoneiros } \\
\hline & & & & & & $\begin{array}{c}N^{o} \\
\text { total }\end{array}$ & Amostra & $\begin{array}{c}N^{o} \\
\text { total }\end{array}$ & Amostra \\
\hline 1 & Leve & $\begin{array}{l}\text { Interno } \\
\text { Externo }\end{array}$ & $\begin{array}{c}6240 \\
\mathrm{~m}^{2} / \mathrm{ano}\end{array}$ & $\begin{array}{c}\text { Piso } \\
\text { Azulejo }\end{array}$ & 38082 & 303 & $\begin{array}{l}\text { (1) G } \\
\text { (10) ADM } \\
\text { (81) O }\end{array}$ & 52 & 26 \\
\hline 2 & Leve & $\begin{array}{l}\text { Interno } \\
\text { Externo }\end{array}$ & $\begin{array}{c}8000000 \\
\mathrm{~m}^{3} / \mathrm{ano}\end{array}$ & $\begin{array}{c}\text { Piso } \\
\text { Azulejo }\end{array}$ & 40000 & 334 & $\begin{array}{l}\text { (2) } \mathrm{G} \\
\text { (16) ADM } \\
\text { (82) O }\end{array}$ & 94 & 47 \\
\hline 3 & Pesada & $\begin{array}{l}\text { Interno } \\
\text { Externo }\end{array}$ & $\begin{array}{l}1150000 \\
\mathrm{~m}^{2} / \mathrm{ano}\end{array}$ & $\begin{array}{l}\text { Perfil de } \\
\text { alumínio }\end{array}$ & 17500 & 416 & $\begin{array}{l}\text { (2) G } \\
\text { (14) ADM } \\
\text { (109) O }\end{array}$ & 28 & 14 \\
\hline
\end{tabular}

Legenda: G - Gerência; ADM - Administrativo; O - Operários; AC - Área Construída; ( ) - № de empregados da amostra. 


\subsection{Resultados Obtidos}

\subsubsection{Perfil socioeconômico dos funcionários e caminhoneiros}

Os dados obtidos em relação ao perfil socioeconômico dos funcionários e caminhoneiros foram: sexo, idade, escolaridade, tipo de vínculo profissional e renda familiar bruta, conforme se apresentam a seguir.

Quanto ao sexo dos funcionários, houve diferença significativa entre o número de homens e mulheres nas três indústrias, com predominância do sexo masculino (84\%) sobre o feminino. Já entre os caminhoneiros, o sexo masculino correspondeu a $100 \%$ da amostra nas três indústrias pesquisadas.

Com relação à faixa etária dos funcionários das indústrias 2 e 3, observa-se que mais de $60 \%$ da amostra têm entre 21 e 30 anos, revelando o perfil de um jovem profissional, seguida pela faixa de 31 a 40 anos. O mesmo não ocorre na indústria 1 , na qual a faixa etária de 31 a 50 anos obteve maior percentual dentre as demais, representando $63,04 \%$ dos pesquisados. A análise da faixa etária de caminhoneiros na indústria 1 mostra uma concentração na faixa de 41 a 50 anos, representando $30,77 \%$ da amostra. Quanto aos caminhoneiros das indústrias 2 e 3, a faixa etária de 31 a 40 anos apresentou maior percentual, $44,68 \%$ e 42,86 respectivamente, seguida pela faixa de 21 a 30 anos.

No que se refere ao nível de escolaridade dos funcionários das indústrias pesquisadas, o percentual relativo ao ensino médio foi superior aos demais, correspondendo a mais de 69\% da amostra. Destaca-se a indústria 2, na qual o nível médio representa $76 \%$, seguido pelo ensino superior: $18 \%$, revelando a política da empresa de contratar profissionais mais qualificados. Quanto à escolaridade dos caminhoneiros, verifica-se, nas indústrias 1 e 2, que o ensino fundamental obteve o maior percentual, $38,46 \%$ e $38,30 \%$ respectivamente. Já na indústria 3, 21,43\% dos pesquisados têm o ensino fundamental completo, com predominância do ensino médio, que totalizou 50\% da amostra analisada.

Com relação à renda familiar bruta dos funcioná- rios, nota-se que $51,09 \%$ dos entrevistados na indústria 1 recebem de 3 a 5 salários mínimos. O mesmo não foi observado nas indústrias 2 e 3 , nas quais houve a predominância da faixa salarial de 1 a 3 salários mínimos. Quanto à renda familiar bruta dos caminhoneiros, observa-se que a maior parte da amostra recebe de 3 a 5 salários mínimos, resultando em um valor superior a $57 \%$ nas três indústrias.

Quando questionados sobre o tipo de vínculo profissional existente, $53,85 \%$ dos caminhoneiros entrevistados na indústria 1 responderam serem autônomos, com destaque para os autônomos contratados, $26,92 \%$. Não se pode desconsiderar o percentual significativo de funcionários assalariados de empresas transportadoras, que correspondeu a $46,15 \%$ da amostra. Nas indústrias 2 e 3, a grande maioria dos entrevistados, $71,43 \%$ da amostra, respondeu ser autônomo, com ênfase para os autônomos agregados de outras empresas. Apenas $28,57 \%$ são funcionários assalariados de empresas transportadoras.

\subsubsection{Características das viagens para funcionários}

Apresenta-se, a seguir, a distribuição modal por indústria pesquisada (ver Tabelas 4 e 5), bem como outras informações relacionadas com as viagens realizadas.

Como se pode observar, há o predomínio da utilização do modo individual nas viagens de acesso às indústrias 1 e 2 . A motocicleta é o meio mais utilizado pelos funcionários, com percentuais de 33,70\% e $50 \%$, respectivamente. Um número significativo de trabalhadores da indústria 1, 30,43\% dos pesquisados, utiliza bicicleta para trabalhar. Segundo a pesquisa, a grande maioria dos funcionários da indústria 3 utiliza o ônibus para ir ao trabalho, totalizando $60 \%$ da amostra. Nota-se o número expressivo de trabalhadores que utilizam a motocicleta como modo de transporte, $20,80 \%$ dos pesquisados. O resultado deve-se ao fato de a indústria 3 estar bem servida quanto ao sistema de transporte coletivo e os funcionários utilizarem o ônibus do tipo fretado; além disso, sua localização é bem próxima a municípios vizinhos como Laguna e

Tabela 4. Modo de transporte (IDA) utilizado pela amostra de funcionários

\begin{tabular}{lcccccc}
\hline \multirow{2}{*}{ Modo de transporte (IDA) } & \multicolumn{2}{c}{ Indústria $\mathbf{1}$} & \multicolumn{2}{c}{ Indústria $\mathbf{2}$} & \multicolumn{2}{c}{ Indústria 3 } \\
\cline { 2 - 7 } & $\boldsymbol{N}^{\boldsymbol{o}}$ func. & $\%$ & $\boldsymbol{N}^{\boldsymbol{o}}$ func. & $\%$ & $\boldsymbol{N}^{\boldsymbol{o}}$ func. & \% \\
\hline Automóvel como motorista & 14 & 15,22 & 15 & 15,00 & 10 & 8,00 \\
\hline Automóvel como passageiro & 2 & 2,17 & 3 & 3,00 & 6 & 4,80 \\
\hline Motocicleta & 31 & 33,70 & 50 & 50,00 & 26 & 20,80 \\
\hline Bicicleta & 28 & 30,43 & 12 & 12,00 & 2 & 1,60 \\
\hline 1 ônibus de linha & 6 & 6,52 & 17 & 17,00 & 68 & 54,40 \\
\hline 2 ou mais ônibus & 4 & 4,35 & 1 & 1,00 & 7 & 5,60 \\
\hline A pé & 7 & 7,61 & 2 & 2,00 & 6 & 4,80 \\
\hline
\end{tabular}


Tabela 5. Modo de transporte (VOLTA) utilizado pela amostra de funcionários

\begin{tabular}{lccccccc}
\hline \multirow{2}{*}{ Modo de transporte (VOLTA) } & \multicolumn{2}{c}{ Indústria $\mathbf{1}$} & \multicolumn{2}{c}{ Indústria $\mathbf{3}$} & \multicolumn{2}{c}{ Indústria 3 } \\
\cline { 2 - 7 } & $\boldsymbol{N}^{\boldsymbol{o}}$ func. & $\boldsymbol{\%}$ & $\boldsymbol{N}^{\boldsymbol{o}}$ func. & $\mathbf{\%}$ & $\boldsymbol{N}^{\boldsymbol{o}}$ func. & $\boldsymbol{\%}$ \\
\hline Automóvel como motorista & 14 & 15,22 & 15 & 15,00 & 10 & 8,00 \\
\hline Automóvel como passageiro & 2 & 2,17 & 3 & 3,00 & 5 & 4,00 \\
\hline Motocicleta & 31 & 33,70 & 50 & 50,00 & 26 & 20,80 \\
\hline Bicicleta & 28 & 30,43 & 12 & 12,00 & 2 & 1,60 \\
\hline 1 ônibus de linha & 6 & 6,52 & 17 & 17,00 & 68 & 54,40 \\
\hline 2 ou mais ônibus & 4 & 4,35 & 1 & 1,00 & 8 & 6,40 \\
\hline A pé & 7 & 7,61 & 2 & 2,00 & 6 & 4,80 \\
\hline
\end{tabular}

Capivari de Baixo, atraindo trabalhadores dessas regiões.

Quanto ao modo de transporte utilizado pelos funcionários na viagem de egresso às indústrias, apenas a indústria 3 apresentou uma pequena alteração nos resultados com relação à viagem de ida, sendo as diferenças encontradas pouco significativas.

Na Tabela 6, são descritos os tempos de viagem dos funcionários, na qual se observa que mais de $88 \%$ das viagens às indústrias 1 e 2 são realizadas em até 30 minutos, com predominância do tempo de até 15 minutos. Na indústria 3, o tempo de viagem até 30 minutos representa $56 \%$ da amostra, embora se note um percentual significativo de viagens realizadas de 46 minutos a 1 hora, $28 \%$ da amostra.

Na Tabela 7, apresentam-se os tempos de viagem de egresso às três indústrias pesquisadas.
Houve uma pequena variação dos resultados encontrados nas viagens de volta realizadas pelos funcionários da indústria 3, quando comparados aos valores de ida, como se observa na Tabela 7 , pois somente um indivíduo passou da condição de passageiro de automóvel a usuário de transporte coletivo.

As cidades de origem e destino dos funcionários são descritas na Tabela 8, na qual se observa que o município sede (Tubarão) apresenta percentual maior que $70 \%$ nas indústrias 1 e 2, explicando os resultados obtidos quanto ao curto tempo de deslocamento nas viagens de ida. Apenas para a indústria 3, este percentual é inferior a $50 \%$, destacando-se também a cidade de Laguna como origem das viagens.

Tabela 6. Tempo de viagem (IDA) da amostra de funcionários

\begin{tabular}{lccccccc}
\hline \multirow{2}{*}{ Tempo de viagem (IDA) } & \multicolumn{2}{c}{ Indústria 1 } & \multicolumn{2}{c}{ Indústria $\mathbf{c}$} & \multicolumn{2}{c}{ Indústria 3 } \\
\cline { 2 - 7 } & $\boldsymbol{N}^{\text {o }}$ func. & $\boldsymbol{\%}$ & $\boldsymbol{N}^{\boldsymbol{o}}$ func. & $\boldsymbol{\%}$ & $\boldsymbol{N}^{\boldsymbol{o}}$ func. & $\boldsymbol{\%}$ \\
\hline$<15 \mathrm{~min}$ & 56 & 60,87 & 57 & 57,00 & 40 & 32,00 \\
\hline $16-30$ minutos & 25 & 27,17 & 35 & 35,00 & 30 & 24,00 \\
\hline $31-45$ minutos & 9 & 9,78 & 5 & 5,00 & 18 & 14,40 \\
\hline 46 minutos -1 hora & 1 & 1,09 & 2 & 2,00 & 35 & 28,00 \\
\hline 1 hora -1 hora e 30 minutos & 1 & 1,09 & 1 & 1,00 & 2 & 1,60 \\
\hline$>1$ hora e 30 minutos & 0 & 0,00 & 0 & 0,00 & 0 & 0,00 \\
\hline
\end{tabular}

Tabela 7. Tempo de viagem (VOLTA) da amostra de funcionários

\begin{tabular}{lccccccc}
\hline \multirow{2}{*}{ Tempo de viagem (VOLTA) } & \multicolumn{2}{c}{ Indústria $\mathbf{1}$} & \multicolumn{2}{c}{ Indústria $\mathbf{2}$} & \multicolumn{2}{c}{ Indústria 3 } \\
\cline { 2 - 7 } & $\boldsymbol{N}^{\boldsymbol{o}}$ func. & $\boldsymbol{\%}$ & $\boldsymbol{N}^{\boldsymbol{o}}$ func. & $\boldsymbol{\%}$ & $\boldsymbol{N}^{\boldsymbol{o}}$ func. & $\boldsymbol{\%}$ \\
\hline$<15 \mathrm{~min}$ & 56 & 60,87 & 57 & 57,00 & 39 & 31,20 \\
\hline $16-30$ minutos & 24 & 26,09 & 35 & 35,00 & 31 & 24,80 \\
\hline $31-45$ minutos & 10 & 10,87 & 5 & 5,00 & 17 & 13,60 \\
\hline 46 minutos -1 hora & 1 & 1,09 & 2 & 2,00 & 36 & 28,80 \\
\hline 1 hora -1 hora e 30 minutos & 1 & 1,09 & 1 & 1,00 & 2 & 1,60 \\
\hline$>1$ hora e 30 minutos & 0 & 0,00 & 0 & 0,00 & 0 & 0,00 \\
\hline
\end{tabular}


Tabela 8. Endereço de origem e destino da viagem da amostra de funcionários

\begin{tabular}{lccccccc}
\hline \multirow{2}{*}{ Endereço da origem e destino } & \multicolumn{2}{c}{ Indústria 1 } & \multicolumn{2}{c}{ Indústria $\mathbf{3}$} & \multicolumn{2}{c}{ Indústria 3 } \\
\cline { 2 - 8 } & $\boldsymbol{N}^{\boldsymbol{o}}$ func. & $\boldsymbol{\%}$ & $\boldsymbol{N}^{\boldsymbol{o}}$ func. & $\boldsymbol{\%}$ & $\boldsymbol{N}^{\boldsymbol{0}}$ func. & $\boldsymbol{\%}$ \\
\hline Tubarão & 83 & 90,22 & 77 & 77,00 & 58 & 46,40 \\
\hline Laguna & 4 & 4,35 & 1 & 1,00 & 40 & 32,00 \\
\hline Capivari de Baixo & 3 & 3,26 & 12 & 12,00 & 12 & 9,60 \\
\hline Gravatal & 1 & 1,09 & - & - & 1 & 0,80 \\
\hline Pedras Grandes & 1 & 1,09 & - & - & - & - \\
\hline Jaguaruna & - & - & 5 & 5,00 & 13 & 10,40 \\
\hline Treze de Maio & - & - & 4 & 4,00 & - & - \\
\hline Urussanga & - & - & 1 & 1,00 & - & - \\
\hline Criciúma & - & - & - & - & 1 & 0,80 \\
\hline
\end{tabular}

\subsubsection{Características das viagens para caminhoneiros}

A seguir, são apresentados os padrões de viagens para os caminhoneiros da indústria em estudo. Quanto ao item freqüência de viagens, observado na Tabela 9, para as indústrias 1 e 2 , os resultados demonstram que mais de $68 \%$ dos motoristas realizaram de 2 a 4 viagens, com ênfase para a freqüência de quatro viagens por mês. Na indústria 3, 14,29\% dos entrevistados responderam terem realizado a viagem pela primeira vez e a predominância ocorre nas freqüências 1 e 4 viagens por mês.

Na Tabela 10, apresentam-se os tempos de viagens dos caminhoneiros desde a cidade de origem até a indústria, com objetivo de carregar o caminhão, em que observa-se que a maioria das viagens ocorre num tempo de até 5 horas.

Tabela 9. Freqüência de viagens da amostra de caminhoneiros

\begin{tabular}{cccccccc}
\hline \multirow{2}{*}{ Freqüência de viagens } & \multicolumn{2}{c}{ Indústria $\mathbf{1}$} & \multicolumn{2}{c}{ Indústria $\mathbf{2}$} & \multicolumn{2}{c}{ Indústria 3 } \\
\cline { 2 - 7 } & $\boldsymbol{N}^{\boldsymbol{o}}$ mot. & $\boldsymbol{\%}$ & $\boldsymbol{N}^{\boldsymbol{o}}$ mot. & $\boldsymbol{\%}$ & $\boldsymbol{N}^{\boldsymbol{o}}$ mot. & $\boldsymbol{\%}$ \\
\hline 1 vez por mês & 4 & 15,38 & 8 & 17,02 & 4 & 28,57 \\
\hline 2 vezes por mês & 8 & 30,77 & 12 & 25,53 & 1 & 7,14 \\
\hline 3 vezes por mês & 1 & 3,85 & 3 & 6,38 & 2 & 14,29 \\
\hline 4 vezes por mês & 10 & 38,46 & 17 & 36,17 & 4 & 28,57 \\
\hline 5 vezes por mês & 0 & 0,00 & 1 & 2,13 & 0 & 0,00 \\
\hline Mais de 5 vezes por mês & 3 & 11,54 & 3 & 6,38 & 1 & 7,14 \\
\hline Primeira vez & 0 & 0,00 & 3 & 6,38 & 2 & 14,29 \\
\hline
\end{tabular}

Tabela 10. Tempo de viagem (IDA) da amostra de caminhoneiros

\begin{tabular}{|c|c|c|c|c|c|c|}
\hline \multirow{2}{*}{ Tempo de viagem (IDA) } & \multicolumn{2}{|c|}{ Indústria 1} & \multicolumn{2}{|c|}{ Indústria 2} & \multicolumn{2}{|c|}{ Indústria 3} \\
\hline & $N^{o}$ mot. & $\%$ & $N^{o}$ mot. & $\%$ & $N^{o}$ mot. & $\%$ \\
\hline$<1$ hora & 15 & 57,69 & 14 & 29,79 & 10 & 71,43 \\
\hline$>1$ hora -3 horas & 1 & 3,85 & 8 & 17,02 & 1 & 7,14 \\
\hline$>3$ horas -5 horas & 5 & 19,23 & 6 & 12,77 & 1 & 7,14 \\
\hline$>5$ horas -7 horas & 0 & 0,00 & 4 & 8,51 & 1 & 7,14 \\
\hline$>7$ horas -9 horas & 2 & 7,69 & 0 & 0,00 & 0 & 0,00 \\
\hline$>9$ horas -11 horas & 0 & 0,00 & 0 & 0,00 & 0 & 0,00 \\
\hline$>11$ horas -13 horas & 0 & 0,00 & 0 & 0,00 & 1 & 7,14 \\
\hline$>13$ horas -15 horas & 1 & 3,85 & 2 & 4,26 & 0 & 0,00 \\
\hline$>15$ horas -17 horas & 0 & 0,00 & 3 & 6,38 & 0 & 0,00 \\
\hline$>17$ horas -19 horas & 0 & 0,00 & 10 & 21,28 & 0 & 0,00 \\
\hline$>19$ horas -24 horas & 1 & 3,85 & 0 & 0,00 & 0 & 0,00 \\
\hline$>24$ horas & 1 & 3,85 & 0 & 0,00 & 0 & 0,00 \\
\hline
\end{tabular}


Tabela 11. Tempo de viagem (VOLTA) da amostra de caminhoneiros

\begin{tabular}{ccccccc}
\hline \multirow{2}{*}{ Tempo de viagem (VOLTA) } & \multicolumn{2}{c}{ Indústria 1 } & \multicolumn{2}{c}{ Indústria $\mathbf{3}$} & \multicolumn{2}{c}{ Indústria 3 } \\
\cline { 2 - 7 } & $\boldsymbol{N}^{\boldsymbol{o}}$ mot. & $\boldsymbol{\%}$ & $\boldsymbol{N}^{\boldsymbol{o}}$ mot. & $\boldsymbol{\%}$ & $\boldsymbol{N}^{\boldsymbol{o}}$ mot. & $\boldsymbol{\%}$ \\
\hline$<1$ hora & 1 & 3,85 & 2 & 4,26 & 2 & 14,29 \\
\hline$>1$ hora -3 horas & 2 & 7,69 & 0 & 0,00 & 0 & 0,00 \\
\hline$>3$ horas -5 horas & 2 & 7,69 & 2 & 4,26 & 3 & 21,43 \\
\hline$>5$ horas -7 horas & 0 & 0,00 & 3 & 6,38 & 1 & 7,14 \\
\hline$>7$ horas -9 horas & 0 & 0,00 & 2 & 4,26 & 0 & 0,00 \\
\hline$>9$ horas -11 horas & 3 & 11,54 & 4 & 8,51 & 0 & 0,00 \\
\hline$>11$ horas -13 horas & 2 & 7,69 & 0 & 0,00 & 1 & 7,14 \\
\hline$>13$ horas -15 horas & 0 & 0,00 & 2 & 4,26 & 4 & 28,57 \\
\hline$>15$ horas -17 horas & 3 & 11,54 & 7 & 14,89 & 0 & 0,00 \\
\hline$>17$ horas -19 horas & 6 & 23,08 & 18 & 38,30 & 1 & 7,14 \\
\hline$>19$ horas -24 horas & 2 & 7,69 & 0 & 0,00 & 0 & 0,00 \\
\hline$>24$ horas & 5 & 19,23 & 7 & 14,89 & 2 & 14,29 \\
\hline
\end{tabular}

As viagens são relativamente curtas para as indústrias 1 e 3, apresentando tempos máximos de até 1 hora, com destaque para a indústria 3 , na qual este período de tempo corresponde a $71,43 \%$ do total de entrevistados. Este resultado deve-se ao fato de os caminhoneiros residirem em cidades próximas à sede da indústria. Na indústria 2, o intervalo de 17 a 19 horas - que é o período de viagem mais longo indicado na pesquisa - representa um percentual significativo de $21,28 \%$ da amostra de caminhoneiros. Os demais intervalos de tempo considerados importantes se situam entre 1 e 5 horas.

São apresentados, na Tabela 11, os tempos de viagem das indústrias até o destino, onde será realizada a entrega do produto.

As viagens de deslocamento dos motoristas das indústrias 1 e 2 são longas, com mais de $60 \%$ das viagens realizadas em mais de 15 horas. Na indústria 3 , pode-se observar que $57,14 \%$ das viagens são realizadas em mais de 11 horas, com predominância do intervalo de tempo de 13 a 15 horas. Nota-se, porém, um valor significativo verificado no intervalo de 3 a 5 horas, correspondendo a $21,43 \%$ da amostra de caminhoneiros.

\subsubsection{Taxas de geração de viagens}

A partir da tabulação das contagens volumétricas de veículos realizadas nos acessos das indústrias, classi- ficadas por tipo de veículo, e do levantamento junto à administração de informações relativas à área total construída (AC) e número de funcionários, elaboraram-se taxas de geração de viagens.

A denominação "veículos em geral" refere-se ao somatório dos fluxos veiculares de caminhões, automóveis, motocicletas e bicicletas. Neste estudo, foram utilizadas duas variáveis explicativas indicadas pela bibliografia existente e pela facilidade na obtenção e fidelidade dos dados: a área bruta construída e o número de funcionários das indústrias.

Verifica-se, na Tabela 12, uma grande variação de resultados quanto às taxas obtidas para automóveis nas três indústrias pesquisadas. Com relação aos caminhões, esta variação foi menor.

Isso pode ser explicado pelas características de viagens encontradas em cada indústria, principalmente quanto aos diferentes modos de transporte utilizados por seus funcionários. Outra característica, como porte do empreendimento (AC), também pode ter exercido influência significativa nos resultados finais, não sendo possível agrupar as indústrias para se estabelecer um padrão de geração de viagens para automóveis e caminhões.

As Tabelas 13 e 14 apresentam os resumos das taxas de geração de viagens nos horários de pico da manhã e da tarde dos empreendimentos.

Tabela 12. Resumo das taxas diárias de geração de viagens

\begin{tabular}{cccccc}
\hline \multirow{2}{*}{ Indústria } & \multicolumn{5}{c}{ Variável explicativa } \\
\cline { 2 - 6 } & Automóvel & Caminhão & Veículos em geral & Automóvel & Veículos em geral \\
\cline { 2 - 6 } & 0,37 & 0,27 & 1,67 & $\mathrm{~N}^{\text {o }}$ de funcionários \\
\hline 1 & 0,95 & 0,47 & 2,47 & 1,14 & 2,96 \\
\hline 2 & 1,36 & 0,32 & 2,83 & 0,57 & 1,19 \\
\hline 3 & & & & &
\end{tabular}


Tabela 14. Resumo das taxas de geração de viagens na hora pico da tarde (empreendimento)

\begin{tabular}{cccccc}
\hline \multirow{2}{*}{ Indústria } & \multicolumn{5}{c}{ Variável explicativa } \\
\cline { 2 - 6 } & Automóvel & Caminhão & Veículos em geral & Automóvel & Veículos em geral \\
\cline { 2 - 6 } & 0,021 & 0,026 & 0,15 & 0,19 \\
\hline 1 & 0,08 & 0,03 & 0,24 & 0,026 & 0,28 \\
\hline 2 & 0,16 & 0,006 & 0,28 & 0,07 & 0,12 \\
\hline 3 & &
\end{tabular}

Tabela 15. Resumo dos horários de pico (empreendimento) e das percentagens de pico horário (PPH)

\begin{tabular}{ccccccc}
\hline \multirow{2}{*}{ Indústria } & \multicolumn{3}{c}{ Pico horário (empreendimento) } & \multicolumn{3}{c}{ PPH (\%) } \\
\cline { 2 - 7 } & Entrando & Saindo & Total & Entrando & Saindo & Total \\
\hline 1 & 06h01 e 07h00 & 14h01 e 15h00 & 06h01 e 07h00 & 19,81 & 15,09 & 16,98 \\
\hline 2 & 07h01 e 08h00 & 17h01 e 18h00 & 07h01 e 08h00 & 21,91 & 13,97 & 11,85 \\
\hline 3 & 07h01 e 08h00 & 17h01 e 18h00 & 07h01 e 08h00 & 20,00 & 18,70 & 10,48 \\
\hline
\end{tabular}

Nota-se a proximidade dos números encontrados para as três indústrias com relação à taxa de geração de viagens na hora pico da manhã para veículos em geral, determinada através da variável área construída. Segundo se observa na Tabela 14, houve diferenças significativas nos valores determinados para as taxas de geração de viagens entre as três indústrias pesquisadas para o pico da tarde.

Na Tabela 15, são descritos o resumo dos horários de pico do empreendimento e suas respectivas percentagens de pico horário (PPH), para veículos em geral, de acordo com cada empreendimento.

Observa-se que, nas indústrias 2 e 3, os horários de pico do empreendimento concentraram-se pela manhã, entre 7h01min e 08h00 (entrando), e pela tarde, entre $17 \mathrm{~h} 01 \mathrm{~min}$ e $18 \mathrm{~h} 00$ (saindo), com valores de pico horário próximos entre si. A indústria 1 apresenta horários de pico de entrada e saída e valores de PPH discrepantes das outras indústrias. As percentagens de pico horário (PPH) entrando são superiores aos valores encontrados para as PPH de saída nas três indústrias pesquisadas.

\subsection{Comparação dos resultados obtidos com estudos existentes}

Nos itens a seguir, realizaram-se as comparações dos resultados obtidos na pesquisa com os dimensionamentos obtidos pelo ITE (2008), CET (1983) e Keefer (1966).

\subsubsection{Viagens por $100 m^{2}$ de $A C$}

- Veículos em geral

A análise da Tabela 16 nos permite observar que os valores obtidos com as equações do ITE e CET representam um número de viagens muito superior aos máximos volumes horários desenvolvidos neste estudo, em média $70 \%$ e $90 \%$, respectivamente. É importante ressaltar que os resultados do ITE e os resultados propostos referem-se ao total de viagens geradas, enquanto os resultados da CET são referentes apenas às viagens atraídas.

\subsubsection{Viagens por número de funcionários}

- Automóveis e caminhões

Os valores de Keefer (1966), utilizados na Tabela 17 , correspondem ao valor médio do total de viagens diárias e/ou na hora pico do empreendimento, no estudo realizado em nove indústrias pesquisadas pelo

Tabela 16. Comparativo de volume de viagens de veículos em geral propostos para as indústrias e os obtidos pelo ITE e pela CET

\begin{tabular}{ccccc}
\hline \multirow{2}{*}{ Indústria } & \multicolumn{2}{c}{ Hora pico da tarde (empreendimento) } & \multicolumn{2}{c}{ Hora pico (empreendimento) } \\
\cline { 2 - 5 } & Proposto & ITE & Proposto & CET \\
\hline 1 & 56 & 411,28 & 108 & $1.156,89$ \\
\hline 2 & 94 & 432,00 & 117 & 52 \\
\hline 3 & 49 & 119 & 518,85 \\
\hline
\end{tabular}

Tabela 17. Comparativo de volume de viagens de automóveis e caminhões, por número de funcionários, propostos para as indústrias e os obtidos por Keefer

\begin{tabular}{|c|c|c|c|c|c|c|c|c|}
\hline \multirow{3}{*}{ Indústria } & \multicolumn{4}{|c|}{ Volume diário } & \multicolumn{4}{|c|}{ Volume hora pico (empreendimento) } \\
\hline & \multicolumn{2}{|c|}{ Proposto } & \multicolumn{2}{|c|}{ Keefer } & \multicolumn{2}{|c|}{ Proposto } & \multicolumn{2}{|c|}{ Keefer } \\
\hline & Auto & Caminhão & Auto & Caminhão & Auto & Caminhão & Auto & Caminhão \\
\hline 1 & 141 & 104 & 558 & 35 & 22 & 5 & 207 & 2 \\
\hline 2 & 381 & 188 & 558 & 35 & 46 & 16 & 207 & 2 \\
\hline 3 & 239 & 56 & 558 & 35 & 32 & 1 & 207 & 2 \\
\hline
\end{tabular}


Tabela 18. Comparativo de volume de viagens de veículos em geral, por número de funcionários, propostos para as indústrias 1,2 e 3 e os obtidos pelo ITE e pela CET

\begin{tabular}{ccccc}
\hline \multirow{2}{*}{ Indústria } & \multicolumn{2}{c}{ Hora pico da tarde (empreendimento) } & \multicolumn{2}{c}{ Hora pico (empreendimento) } \\
\cline { 2 - 5 } & Proposto & ITE & Proposto & CET \\
\hline 1 & 56 & 154,53 & 108 & 152,96 \\
\hline 2 & 94 & 170,34 & 117 & 52 \\
\hline 3
\end{tabular}

autor.

Ressalta-se que não foram calculadas na pesquisa as taxas de geração de viagens de caminhões, para cada indústria em função da variável explicativa " $\mathrm{N}^{0}$ de funcionários”, já que Keefer (1966) divulgou em seu trabalho apenas os volumes obtidos. Para fins comparativos serão utilizados os volumes de viagens realizadas por caminhões resultantes das contagens volumétricas.

Há uma discrepância entre os volumes de automóvel obtidos por Keefer e os propostos na pesquisa, explicados pelas diferenças entre a realidade brasileira e a americana, na qual o automóvel é amplamente utilizado nas viagens às indústrias. Já nas viagens de caminhões, apenas o volume na hora pico do empreendimento proposto na pesquisa (indústria 3) apresentou resultados superiores aos obtidos pelo autor, na ordem de $37,5 \%$. Os demais volumes propostos são inferiores em média $70 \%$.

- Veículos em geral

Assim como observado nos demais itens, os volumes apresentados pelo ITE e CET, utilizando-se a variável número de funcionários, também são superiores ao máximo volume horário obtido na pesquisa, em média $65 \%$ e $45 \%$.

\section{CONCLUSÕES}

Através da tabulação dos dados, chegou-se ao perfil do industriário: um profissional jovem, do sexo masculino, com renda média entre 1 a 3 salários mínimos e nível médio de escolaridade. Observa-se uma equivalência entre os meios de transporte utilizados para realização das viagens de ida e volta ao trabalho entre automóveis, motocicletas e transporte coletivo. As viagens são curtas, prevalecem os intervalos de tempo de até 15 minutos.

Os motoristas são profissionais autônomos, do sexo masculino, com renda média de 3 a 5 salários mínimos e idade que varia de 31 a 50 anos. Realizam viagens curtas até a indústria e longas para o destino da carga.

Ao serem agrupados os dados referentes à geração de viagens nas três indústrias pesquisadas, nota-se que o comportamento é distinto para cada indústria, principalmente quanto às taxas obtidas por automóveis. Quanto às taxas calculadas na hora pico da manhã (empreendimento) para veículos em geral, utilizando- se a variável área construída, constatou-se a proximidade dos números encontrados nas três indústrias, igualmente verificado em relação às taxas diárias geradas por caminhões.

Características como atividades desenvolvidas em cada empreendimento, porte e número de funcionários distintos resultaram em diferentes taxas de geração, dificultando a obtenção de um padrão de geração de viagens para indústrias. Os diferentes modais também podem ter sido determinantes para a variação das taxas, tendo a motocicleta obtido um percentual de $33,70 \%$ e de $50 \%$ nas indústrias 1 e 2 respectivamente, e o ônibus 60,80\% na indústria 3 .

Comparando-se as taxas e os volumes de viagens estipulados pelo ITE (2008), por Keefer (1966) e pela CET-SP (1983) com os encontrados neste estudo, verifica-se que estes últimos se mostraram inferiores aos dos modelos existentes, exceto o volume diário proposto para caminhões, que apresentou resultados superiores aos dos modelos obtidos por Keefer (1966).

Para indústrias de Tubarão, sugere-se a utilização dos parâmetros definidos nesta pesquisa, levando-se em consideração a semelhança da indústria em questão com uma das três estudadas, já que não foi possível a padronização das taxas obtidas. Também sugerese a ampliação deste tipo de estudo em outras cidades brasileiras, pois os resultados obtidos apresentaram realidades distintas. Ressalta-se que a extensão do estudo e o agrupamento das indústrias por tipo de atividade permitirão a obtenção de valores mais abrangentes para as taxas de geração de viagens.

Embora não passíveis de uma padronização, os levantamentos realizados e seus resultados significam uma contribuição importante para o planejamento adequado do sistema viário e de transportes relacionado às indústrias. Apesar de terem apresentado a realidade de uma única cidade, representam o embrião ao estudo desse tipo de empreendimento no Brasil, sendo úteis para a ampliação e o fortalecimento das pesquisas desenvolvidas pela Rede Ibero-Americana de Estudos em Pólos Geradores de Viagens.

\section{REFERÊNCIAS BIBLIOGRÁFICAS}

Carlos, A. F. A. (1990) Espaço e Indústria. Editora Contexto. São Paulo. CET- Companhia de Engenharia de Tráfego (1983) Pólos Geradores de Tráfego, Boletim Técnico n³2, São Paulo.

ITE - Institute of Transportation Engineers (2008) Trip Generation, 8th edition, Washington DC. 
Keefer, L. E. (1966) Urban travel patterns for airports, shopping centers, and industrial plants. NCHRP, Report 24, Highway Research Board.

Pitombo, C. S; E. Kawamoto e J. A. Quintanilha (2006) Investigação da influência do uso do solo e das características socioeconômicas na seqüência de deslocamentos dos industriários na Região Metropolitana de São Paulo. Anais do XX Congresso de pesquisa e Ensino em Transportes, ANPET, Brasília-DF. 